\title{
IMPROVEMENTS OF SOME INTEGRAL INEQUALITIES OF GRÜSS TYPE
}

\author{
J. PEČARIĆ AND B. TEPEŠ
}

S. S. Dragomir ([1]) has proved the following results:

Theorem A. Let $f, g:[a, b] \rightarrow \boldsymbol{R}$ be two differentiable mappings on $(a, b)$. If $f^{\prime} \in L_{\alpha}(a, b)$ and $g^{\prime} \in L_{\beta}(a, b)$ with $\alpha>1$ and $\frac{1}{\alpha}+\frac{1}{\beta}=1$, then we have the inequality

$$
\begin{aligned}
|T(f, g)| \leq & \frac{1}{2}\left(\left.\frac{1}{(b-a)^{2}} \int_{a}^{b} \int_{a}^{b}|x-y|\left|\int_{x}^{y}\right| f^{\prime}(t)\right|^{\alpha} d t \mid d x d y\right)^{\frac{1}{\alpha}} \\
& \times\left(\left.\frac{1}{(b-a)^{2}} \int_{a}^{b} \int_{a}^{b}|x-y|\left|\int_{x}^{y}\right| g^{\prime}(t)\right|^{\beta} d t \mid d x d y\right)^{\frac{1}{\beta}} \\
\leq & \frac{1}{6}\left\|f^{\prime}\right\|_{\alpha}\left\|g^{\prime}\right\|_{\beta}(b-a) .
\end{aligned}
$$

where

$$
T(f, g)=\frac{1}{b-a} \int_{a}^{b} f(x) g(x) d x-\frac{1}{b-a} \int_{a}^{b} f(x) d x \cdot \frac{1}{b-a} \int_{a}^{b} g(x) d x .
$$

The first inequality in (1) is sharp.

Theorem B. Let $f, g:[a, b] \rightarrow \boldsymbol{R}$ be two differentiable mappings on $(a, b)$. If $f^{\prime} \in L_{\infty}(a, b)$ and $g^{\prime} \in L_{1}(a, b)$ then we have the inequality

$$
\begin{aligned}
|T(f, g)| & \leq \frac{1}{2(b-a)^{2}} \int_{a}^{b} \int_{a}^{b}|x-y| \sup _{t \in[x, y]}\left|f^{\prime}(t)\right|\left|\int_{x}^{y}\right| g^{\prime}(z)|d z| d x d y \\
& \leq \frac{1}{6}\left\|f^{\prime}\right\|_{\infty}\left\|g^{\prime}\right\|_{1}(b-a) .
\end{aligned}
$$

The first inequality in (2) is sharp.

In this paper we shall improve the second inequalities in (1) and (2), i.e. the following theorem is valid:

Received August 11, 2003. 
Theorem. (i) Let the assumptions of Theorem A be fulfiled. Then

$$
\begin{aligned}
|T(f, g)| \leq & \frac{1}{2}\left(\left.\frac{1}{(b-a)^{2}} \int_{a}^{b} \int_{a}^{b}|x-y|\left|\int_{x}^{y}\right| f^{\prime}(t)\right|^{\alpha} d t \mid d x d y\right)^{\frac{1}{\alpha}} \\
& \times\left(\left.\frac{1}{(b-a)^{2}} \int_{a}^{b} \int_{a}^{b}|x-y|\left|\int_{x}^{y}\right| g^{\prime}(t)\right|^{\beta} d t \mid d x d y\right)^{\frac{1}{\beta}} \\
\leq & \frac{1}{8}\left\|f^{\prime}\right\|_{\alpha}\left\|g^{\prime}\right\|_{\beta}(b-a) .
\end{aligned}
$$

(ii) Let the assumptions os Theorem $B$ be fulfilled. Then

$$
\begin{aligned}
|T(f, g)| & \leq \frac{1}{2(b-a)^{2}} \int_{a}^{b} \int_{a}^{b}|x-y| \sup _{t \in[x, y]}\left|f^{\prime}(t)\right|\left|\int_{x}^{y}\right| g^{\prime}(t)|d t| d x d y \\
& \leq \frac{1}{8}\left\|f^{\prime}\right\|_{\infty}\left\|g^{\prime}\right\|_{1}(b-a) .
\end{aligned}
$$

Proof. (i) Let us consider the integral

$$
\begin{aligned}
\int_{a}^{b} \int_{a}^{b} & \left.|x-y|\left|\int_{x}^{y}\right| f^{\prime}(t)\right|^{\alpha} d t \mid d x d y \\
& =2 \int_{a}^{b} \int_{a}^{x}(x-y) \int_{x}^{y}\left|f^{\prime}(t)\right|^{\alpha} d t d y d x \\
& =2 \int_{a}^{b} \int_{a}^{x} \int_{a}^{t}(x-y)\left|f^{\prime}(t)\right|^{\alpha} d y d t d x \\
& =\int_{a}^{b} \int_{a}^{x}\left[(x-a)^{2}-(x-t)^{2}\right]\left|f^{\prime}(t)\right|^{\alpha} d t d x \\
& =\int_{a}^{b}\left|f^{\prime}(t)\right|^{\alpha} \int_{t}^{b}\left[(x-a)^{2}-(x-t)^{2}\right] d x d t \\
& =\frac{1}{3} \int_{a}^{b}\left[(b-a)^{3}-(t-a)^{3}-(b-t)^{3}\right]\left|f^{\prime}(t)\right|^{\alpha} d t
\end{aligned}
$$

By elementary calculus we can obtain

$$
(b-a)^{3}-(t-a)^{3}-(b-t)^{3} \leq \frac{3}{4}(b-a)^{3} .
$$

Therefore, we have

$$
\left.\left.\int_{a}^{b} \int_{a}^{b}|x-y|\left|\int_{x}^{y}\right| f^{\prime}(t)\right|^{\alpha} d t\left|d x d y \leq \frac{1}{4}(b-a)^{3} \int_{a}^{b}\right| f^{\prime}(t)\right|^{\alpha} d t=\frac{1}{4}(b-a)^{3}\left\|f^{\prime}\right\|_{\alpha}^{\alpha} .
$$

It is clear that we also have

$$
\left.\left.\int_{a}^{b} \int_{a}^{b}|x-y|\left|\int_{x}^{y}\right| g^{\prime}(t)\right|^{\beta} d t\left|d x d y \leq \frac{1}{4}(b-a)^{3} \int_{a}^{b}\right| g^{\prime}(t)\right|^{\beta} d t=\frac{1}{4}(b-a)^{3}\left\|g^{\prime}\right\|_{\beta}^{\beta} .
$$


Therefore, we have

$$
\begin{aligned}
\frac{1}{2}\left(\left.\frac{1}{(b-a)^{2}} \int_{a}^{b} \int_{a}^{b}|x-y|\left|\int_{x}^{y}\right| f^{\prime}(t)\right|^{\alpha} d t \mid d x d y\right)^{\frac{1}{\alpha}} \\
\quad \times\left(\left.\frac{1}{(b-a)^{2}} \int_{a}^{b} \int_{a}^{b}|x-y|\left|\int_{x}^{y}\right| g^{\prime}(t)\right|^{\beta} d t \mid d x d y\right)^{\frac{1}{\beta}} \\
\leq \frac{1}{2}\left(\frac{1}{4}(b-a)\left\|f^{\prime}\right\|_{\alpha}^{\alpha}\right)^{\frac{1}{\alpha}}\left(\frac{1}{4}(b-a)\left\|g^{\prime}\right\|_{\beta}^{\beta}\right)^{\frac{1}{\beta}} \\
=\frac{1}{8}\left\|f^{\prime}\right\|_{\alpha}\left\|g^{\prime}\right\|_{\beta}(b-a) .
\end{aligned}
$$

(ii) Similarly, we can obtain

$$
\begin{aligned}
\int_{a}^{b} \int_{a}^{b} & |x-y| \sup _{t \in[x, y]}\left|f^{\prime}(t)\right|\left|\int_{x}^{y}\right| g^{\prime}(t)|d t| d x d y \\
& \leq\left\|f^{\prime}\right\|_{\infty} \int_{a}^{b} \int_{a}^{b}|x-y|\left|\int_{x}^{y}\right| g^{\prime}(t)|d t| d x d y \\
& \leq \frac{1}{4}\left\|f^{\prime}\right\|_{\infty}\left\|g^{\prime}\right\|_{1}(b-a)^{3}
\end{aligned}
$$

i.e. we have improvement of the second inequality in (2), that is

$$
\frac{1}{2(b-a)^{2}} \int_{a}^{b} \int_{a}^{b}|x-y| \sup _{t \in[x, y]}\left|f^{\prime}(t)\right|\left|\int_{x}^{y}\right| g^{\prime}(t)|d t| d x d y \leq \frac{1}{8}\left\|f^{\prime}\right\|_{\infty}\left\|g^{\prime}\right\|_{1}(b-a) .
$$

Remark. It was prove in [2] (see also [3, p.202]):

$$
|T(f, g)| \leq \frac{b-a}{4}\left[\frac{2^{\alpha}-1}{\alpha(\alpha+1)}\right]^{\frac{1}{\alpha}}\left[\frac{2^{\beta}-1}{\beta(\beta+1)}\right]^{\frac{1}{\beta}}\left\|f^{\prime}\right\|_{\alpha}\left\|g^{\prime}\right\|_{\beta}
$$

if $f^{\prime} \in L_{\alpha}, g^{\prime} \in L_{\beta}, \alpha \geq 1, \frac{1}{\alpha}+\frac{1}{\beta}=1$.

For $\alpha=\beta=2$ we have

$$
|T(f, g)| \leq \frac{b-a}{8}\left\|f^{\prime}\right\|_{2}\left\|g^{\prime}\right\|_{2},
$$

while for $\alpha=1, \beta=\infty$ we get

$$
|T(f, g)| \leq \frac{b-a}{4}\left\|f^{\prime}\right\|_{1}\left\|g^{\prime}\right\|_{\infty}
$$

Results obtained in this paper show that constant $\frac{1}{8}$ is valid for all $\alpha, 1 \leq \alpha \leq \infty$. On the other hand side it is well known that inequality (3) can be improved. Namely, A. Lupaş [4] has proved that

$$
|T(f, g)| \leq \frac{b-a}{\pi^{2}}\left\|f^{\prime}\right\|_{2}\left\|f^{\prime}\right\|_{2}
$$




\section{References}

[1] S. S. Dragomir, Some inequalities of Grüss type, Indian J. Pure Appl. Math. 31(2000), 397-415.

[2] P. R. Beesack, D. S. Mitrinović and P. M. Vasić, Integral inequalities (the manuscript not achivied and not publiched).

[3] D. S. Mitrinović, J. E. Pečarić and A. M. Fink, Classical and New Inequalities in Analysis, Kluwer Academic Publishers, Dordrecht, 1993.

[4] A. Lupaş, The best constant in an integral inequality, Mathematica 15(1973), 219-222.

Faculty of Textile Technology, University of Zagreb, Pierottijeva 6, 10000 Zagreb, Croatia.

E-mail: pecaric@hazu.hr

Faculty of Philosophy, Dept. of Information. Sci., University of Zagreb, I. Lučića 3, 10000 Zagreb, Croatia.

E-mail: btepes@ffzg.hr 